\title{
Émission $X(L)$ du xénon par interaction laser-agrégats
}

\author{
L. Adoui ${ }^{1}$, O. Gobert ${ }^{3}$, P. Indelicato ${ }^{2}$, E. Lamour, P. Meynadier ${ }^{3}$, D. Normand ${ }^{3}$, \\ M. Perdrix ${ }^{3}$, C. Prigent, J.-P. Rozet et D. Vernhet \\ GPS-PIIM, Universités Paris VI et Paris VII, 2 place Jussieu, 75251 Paris cedex 05, France \\ ${ }^{1}$ CIRIL, rue Claude Bloch, BP. 5133, 14070 Caen cedex 5, France \\ ${ }^{2}$ LKB, ENS et Université Paris VI, 4 place Jussieu, 75252 Paris cedex 05, France \\ ${ }^{3}$ CEA-Saclay, DSM/DRECAM/SPAM, 91191 Gif-sur-Yvette cedex, France
}

\begin{abstract}
Résumé: Nous avons étudié le rayonnement $X$ provenant d'ions fortement multichargés $(\geq 24+)$ présentant des lacunes en couche $L$ produits lors de l'irradiation d'agrégats de xénon par des impulsions lumineuses issues d'un laser femtoseconde de puissance. Les résultats obtenus lors de la toute dernière campagne d'expériences réalisée auprès du serveur LUCA du SPAM/DRECAM au CEA/Saclay mettent en cause certains travaux antérieurs [4-6]. Des divergences marquées apparaissent tant au niveau de l'interprétation des spectres $\mathrm{X}$ que sur la variation du taux d'émission en fonction de l'éclairement et de la longueur d'onde.
\end{abstract}

\section{INTRODUCTION}

De nombreuses études expérimentales ont montré qu'il était possible d'observer l'émission de photons X dans la gamme $1-5 \mathrm{keV}$ lors de l'irradiation d'agrégats de xénon avec un laser femtoseconde de puissance [1-2]. Tout comme lors de l'interaction d'un tel laser avec des agrégats d'argon [3], le rayonnement $X$ issu d'agrégats de xénon provient d'ions fortement multichargés (avec ici $q \geq 24+$ ) et présentant des lacunes en couche interne (couche $L$ et $M$ ). Des travaux expérimentaux antérieurs [4] ont montré une forte dépendance de l'intensité des $X(L)$ issus d'agrégats de xénon en fonction de la longueur d'onde $\left(\propto \lambda^{-6}\right)$. Ce phénomène est attribué, selon les auteurs, à une forte augmentation du couplage entre les électrons lors de leur mouvement collectif et cohérent responsable de la création de multiples lacunes en couche interne lorsque la longueur d'onde du champ excitateur diminue [5-6]. Après avoir étudié les $\mathrm{X}(\mathrm{K})$ issus d'agrégats d'argon de façon précise [3 et 7], nous avons donc également entrepris d'étudier le rayonnement $\mathrm{X}(\mathrm{L})$ issu d'agrégats de xénon soumis à l'irradiation d'un pulse laser femtoseconde. Les mécanismes de production de lacunes en couche interne, et notamment le mode de chauffage des électrons, restant très controversés à l'heure actuelle, des mesures quantitatives précises en fonction des différents paramètres gouvernant l'interaction sont indispensables afin de tester les différents processus pris en compte dans les modèles existants. Nous avons donc étudié par spectroscopie de haute résolution les $X(L)$ du xénon et déterminé la distribution d'état de charge et son évolution en fonction de l'éclairement laser, la longueur d'onde et la taille des agrégats.

\section{RESULTATS}

Le dispositif expérimental sur lequel nous avons effectué nos campagnes d'expériences a déjà été décrit dans des articles précédents [ 3 et 7]. Brièvement, un jet pulsé d'agrégats, produit par expansion adiabatique d'un gaz rare sous pression, est irradié par une impulsion laser femtoseconde (de l'ordre de $60 \mathrm{fs}$ ). L'éclairement laser atteint à l'aide de ce dispositif est compris entre $10^{15} \mathrm{~W} / \mathrm{cm}^{2}$ et $10^{17} \mathrm{~W} / \mathrm{cm}^{2}$ et la taille des agrégats est contrôlée par la pression $\mathrm{P}_{0}$ en amont de la buse conique produisant les agrégats. Le système de détection $X$, calibré en absolu, nous permet non seulement d'étudier la spectroscopie du rayonnement (nature des transitions et distribution ionique dont il est issu), mais également de déterminer le taux de photons $X$ émis dans $4 \pi$ lors de l'interaction.

A l'aide des détecteurs $\mathrm{Si}(\mathrm{Li})$ (spectroscopie basse résolution), le type de lacune créée en couche interne a été identifié par la mesure de 1'énergie moyenne des photons $X$ émis : $\overline{\mathrm{E}} \sim 4,4 \mathrm{keV}$ soit $\lambda \sim 2,8 \AA$. Cette énergie correspond typiquement à des $X(L)$ du xénon. Grâce au spectromètre cristallin de grande transmission et de haute résolution [3], une spectroscopie fine du rayonnement $X$ émis permet de 
suivre quantitativement la distribution d'état de charge des ions émetteurs et d'identifier plus précisément le type de transitions responsables de l'émission $\mathrm{X}$.

Les spectres observés, reportés sur la figure 1, montrent clairement deux groupes distincts de raies X. Ces groupes de raies, également observés dans des études antérieures [4], correspondent en fait chacun à la même distribution d'état de charge des ions $\mathrm{Xe}^{\mathrm{q}^{+}}$émetteurs mais désexcitant des niveaux différents. Kondo et al. [4] ont identifié chacun de ses groupes comme étant des transitions $3 \mathrm{~d} \rightarrow 2 \mathrm{p}$ avec soit une lacune en couche $2 p\left(2 p^{-1}\right)$ soit 2 lacunes en couche $2 p\left(2 p^{-2}\right)$. Des calculs d'énergie de transitions, de type multiconfiguration Hartree - Fock, montrent que l'écart en énergie entre $2 \mathrm{p}^{-1}$ et $2 \mathrm{p}^{-2}$ est totalement incompatible avec l'observation. L'écart $3 \mathrm{~d} \rightarrow 2 \mathrm{p}^{-1} / 3 \mathrm{~d} \rightarrow 2 \mathrm{p}^{-2}$ correspond en effet à environ $120 \mathrm{eV}$ alors que l'écart observé entre les groupes de raies correspond à plus de $300 \mathrm{eV}$. On peut par contre attribuer à chacun de ces groupes de raies une configuration distincte à partir de l'écart de structure fine du niveau $2 \mathrm{p}^{-1}$. En effet, si on considère en première approximation que chacun des 2 groupes de raies observées sont principalement dus à la désexcitation du niveau $3 \mathrm{~d}$ pour différents états de charge de l'ion émetteur Xe $\mathrm{q}^{+}$, l'écart type mesuré correspond à l'écart entre $3 \mathrm{~d}_{3 / 2} \rightarrow 2 \mathrm{p}_{1 / 2}$ et $3 \mathrm{~d}_{5 / 2} \rightarrow 2 \mathrm{p}_{3 / 2}$ soit autour de $310 \mathrm{eV}$ pour des ions d'état de charge supérieur à $24+$ pour les agrégats les plus petits (soit à une pression amont $\mathrm{P}_{0}=3,1$ bar, $c f$. figure 1a). On notera néanmoins qu'une interprétation plus poussée de ces spectres est nécessaire et des calculs multiconfigurationnels sont en cours. En effet, les différentes composantes de structure fine de la transition $3 l_{j}-2 l_{j}$ sont à prendre en compte et un calcul précis des rapports de branchement et des taux Auger / radiatifs est à effectuer pour chaque état de charge. Cependant, les premiers calculs réalisés permettent d'identifier chacun des groupes comme étant du à l'écart de structure fine $2 \mathrm{p}_{1 / 2}-2 \mathrm{p}_{3 / 2}$ et pas à l'écart correspondant à la présence d'une ou deux lacunes en couche $2 \mathrm{p}\left(2 \mathrm{p}^{-1}-\right.$ $2 \mathrm{p}^{-2}$ ).

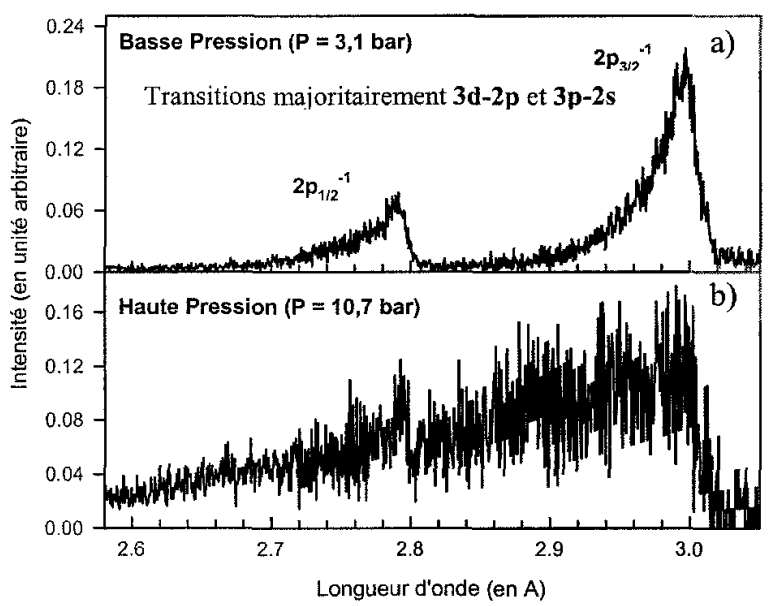

Figure 1. Spectres haute résolution du rayomement $\mathrm{X}$ issu d'agrégats de xénon soumis à une impulsion laser IR de 60 fs et un éclairement crête de l'ordre de $2,9 \cdot 10^{16} \mathrm{~W} / \mathrm{cm}^{2}$ pour une pression amont de a) $P_{0}=3,1$ bar et $b$ ) $P_{0}=10,7$ bar.

L'évolution de ces spectres a été examinée en fonction de la taille des agrégats, de l'éclairement ainsi que pour deux longueurs d'onde laser IR ou UV de façon à pouvoir discuter clairement leur dépendance.

Sur la figure $1 \mathrm{~b}$ est reporté le spectre $\mathrm{X}$ obtenu pour un même éclairement (en IR) que le spectre de la figure 1a, mais pour des agrégats de taille plus importante : la pression en amont du jet a été augmentée de plus d'un facteur 3 ce qui correspond à des agrégats environ 10 fois plus gros dans le cas de la figure $1 \mathrm{~b}$ que ceux de la figure la. On observe clairement une «déformation » de chacun des 2 groupes de raies vers les basses longueurs d'onde, soit les grandes énergies quand on augmente la pression amont. D'autre part, le taux global d'émission $\mathrm{X}(\mathrm{L})$ augmente avec la taille des agrégats suivant une loi en $\mathrm{P}_{0}{ }^{\alpha}$ (avec $\alpha \geq 5 / 2$ d'après une analyse préliminaire). Cet effet se traduit ici par une augmentation du taux d'X d'un facteur 20 entre chacune des pressions étudiées ici; effet qui a été complètement négligé dans les études antérieures [4 et 6$]$. 
Une étude spécifique concernant l'évolution du taux d'X en fonction de l'éclairement laser a été réalisée dans le cas des agrégats de xénon. D'une manière tout à fait similaire à ce qui a été observé pour des agrégats d'argon, les résultats obtenus montrent que le taux d'X à grand éclairement suit une loi en $\mathrm{I}^{3 / 2}$ caractéristique d'une augmentation du volume focal effectif [3]. Cette loi de variation, qui est observée tant en IR qu'en UV, montre que la loi linéaire en énergie laser utilisée par Kondo et al. [4] pour calibrer leur spectre entre lumière IR et UV est incorrecte. Le seuil de production $X$ a été déterminé en $\mathbb{R}$ et apparaît à un éclairement laser très faible : entre 1,5 et $2.10^{15} \mathrm{~W} / \mathrm{cm}^{2}$.

Afin de pouvoir finaliser l'étude comparative du taux d'émission $\mathrm{X}$ et des espèces ioniques produites dans le cas des agrégats de xénon, nous présentons figure 2 les spectres obtenus pour une longueur d'onde de $400 \mathrm{~nm}$ à un éclairement laser identique à celui de la figure 1 et pour deux tailles d'agrégats différentes. Ces pressions sont néanmoins similaires à celles présentés dans le cas de l'étude en $\mathbb{R}$. Nous observons là aussi clairement deux groupes de raies qui sont caractéristiques, par leur différence en énergie, de l'écart de structure fine $2 p_{1 / 2}-2 p_{3 / 2}$. De plus, tout comme pour l'IR, on notera une augmentation de l'état de charge moyen quand la taille des agrégats augmente et qui est caractérisée par un déplacement des deux groupes de raies vers les basses longueurs d'onde (soit les hautes énergies).

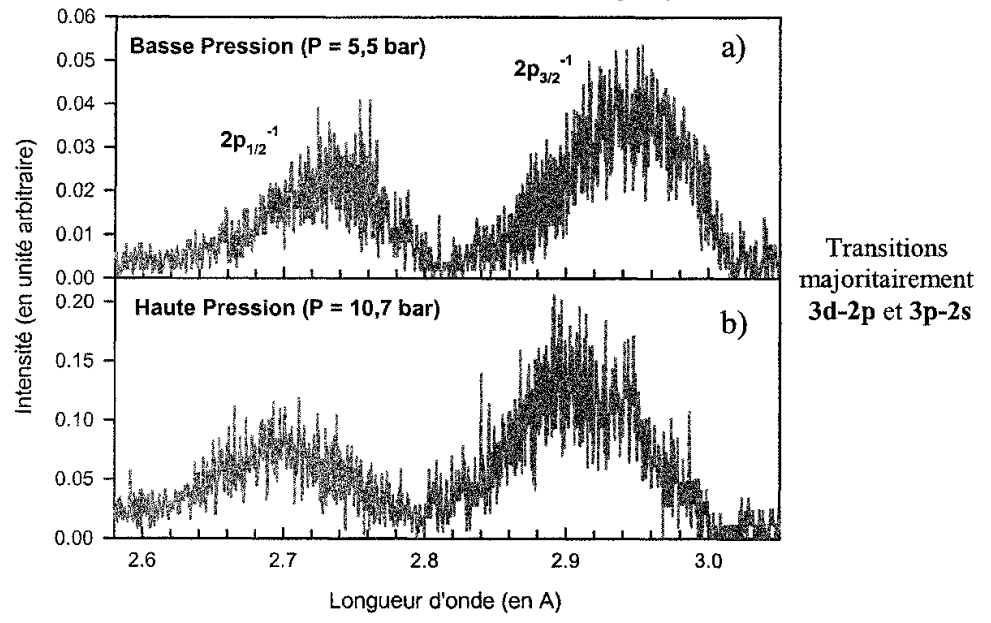

Figure 2. Spectres haute résolution du rayonnement $X$ issu d'agrégats de xénon soumis à une impulsion laser UV de $180 \mathrm{fs}$ et un éclairement de $1^{\prime}$ ordre de $3,0.10^{16} \mathrm{~W} / \mathrm{cm}^{2}$ pour une pression amont de a) $P_{0}=5,5$ bar et b) $P_{0}=10,7$ bar.

En comparant maintenant les résultats obtenus en UV à ceux obtenus en IR pour un éclairement laser équivalent et pour des pressions amonts du jet comparables, on peut remarquer à partir de ces spectres :

i) que l'état de charge moyen en UV à basse pression est plus élevée qu'en IR et que la distribution d'état de charge n'a pas du tout la même forme. Les espèces ioniques créées ne sont pas les mêmes et l'examen détaillé du taux d'épluchage de la couche $M$ (responsable de ces distributions d'état de charge) dans chacun des cas devrait fournir des informations précises sur la compétition entre processus de chauffage et tout réarrangement dont la désexcitation.

ii) que le taux d'émission $X$ à taille d'agrégats identique (soit en comparant figure $1 \mathrm{~b}$ et $2 b$ ) est équivalent voire très légèrement supérieure en UV par rapport à l'IR. Ce résultat est en complet désaccord avec une dépendance en $\lambda^{-6}$ observée par Kondo et al. [4] et modélisé par AndreasSchroeder et al. [6].

\section{CONCLUSION}

L'étude rigoureuse menée ici, dans le cas des agrégats de xénon, sur une même source laser, en maîtrisant les différents paramètres qui jouent sur l'interaction, met en défaut les observations et modélisation antérieures [4 et 6]. L'intensité et la longueur d'onde du laser ainsi que la taille des agrégats influent nettement sur le taux d'émission $X$ et l'état de charge des espèces ioniques créées lors de l'interaction. Néanmoins, à taille d'agrégat et éclairement équivalents, le taux d'émission $\mathrm{X}(\mathrm{L})$ des agrégats de xénon 
ne varie pas en $\lambda^{-6}$ entre IR et UV comme l'on déduit de leurs études Kondo et al. [4]. Il n'en reste pas moins que les distributions d'état de charge des ions xénon, à même conditions, sont très différentes en fonction de la longueur d'onde et un examen approfondi de l'évolution du taux d'épluchage de la couche $M$ responsable de cet effet devrait permettre de quantifier la compétition entre processus de chauffage et réarrangement des électrons sur les couches externes au cours de l'interaction.

\section{Références}

1. Boyer et al., J. Phys. B, 27 (1994) 4373

2. McPherson et al., Nature, 370 (1994) 631

3. Rozet et al., Physica Scripta T92 (2001) 113

4. Kondo et al., J. Phys. B, 30 (1997) 2707

5. Thompson et al., J. Phys. B, 27 (1994) 4391

6. Andreas Schroeder et al., J. Phys. B, 34 (2001) 297

7. Prigent et al., Journal de Physique IV (2002) Proceedings of UVX2002 